\title{
Types on stable Banach spaces
}

\author{
by
}

José I o v i no (Pittsburgh, Penn.)

\begin{abstract}
We prove a geometric characterization of Banach space stability. We show that a Banach space $X$ is stable if and only if the following condition holds. Whenever $\widehat{X}$ is an ultrapower of $X$ and $B$ is a ball in $\widehat{X}$, the intersection $B \cap X$ can be uniformly approximated by finite unions and intersections of balls in $X$; furthermore, the radius of these balls can be taken arbitrarily close to the radius of $B$, and the norm of their centers arbitrarily close to the norm of the center of $B$.

The preceding condition can be rephrased without any reference to ultrapowers, in the language of types, as follows. Whenever $\tau$ is a type of $X$, the set $\tau^{-1}[0, r]$ can be uniformly approximated by finite unions and intersections of balls in $X$; furthermore, the radius of these balls can be taken arbitrarily close to $r$, and the norm of their centers arbitrarily close to $\tau(0)$.

We also provide a geometric characterization of the real-valued functions which satisfy the above condition.
\end{abstract}

1. Introduction. A separable Banach space $X$ is stable if whenever $\left(a_{m}\right)$ and $\left(b_{n}\right)$ are bounded sequences in $X$ and $\mathcal{U}, \mathcal{V}$ are ultrafilters on $\mathbb{N}$,

$$
\lim _{\mathcal{U}, m} \lim _{\mathcal{V}, n}\left\|a_{m}+b_{n}\right\|=\lim _{\mathcal{V}, n} \lim _{\mathcal{U}, m}\left\|a_{m}+b_{n}\right\| .
$$

This concept was introduced by J.-L. Krivine and B. Maurey in [5], where the authors proved that every stable Banach space contains almost isometric copies of $\ell_{p}$, for some $1 \leq p<\infty$. This generalized a result of $\mathrm{D}$. Aldous [1] about subspaces of $L_{1}$.

The concept of type on a Banach space was introduced in [5] as well. If $X$ is a Banach space and $a \in X$, the type realized by $a$ is the function $\tau_{a}: X \rightarrow \mathbb{R}$ defined by $\tau_{a}(x)=\|x+a\|$. The space of types of $X$, denoted by $\mathcal{T}(X)$, is the closure of $\left\{\tau_{a} \mid a \in X\right\}$ in $\mathbb{R}^{X}$ with respect to the product topology. The norm of a type $\tau$ is $\tau(0)$.

1991 Mathematics Subject Classification: Primary 46B20; Secondary 46B08, 46B07, $46 \mathrm{~B} 20$.

The author would like to express his gratitude to C. W. Henson for several valuable conversations. 
The role played by types in [5] generalizes that played by random measures in [1].

Since [5], stable Banach spaces and types have been studied intensely. For a self-contained exposition, we refer the reader to [2].

Types can be viewed quite naturally in terms of Banach space ultrapowers as follows. A type on $X$ is a function $\tau: X \rightarrow \mathbb{R}$ such that there exists an ultrapower $\widehat{X}$ of $X$ and an element $a \in \widehat{X}$ with

$$
\tau(x)=\|x+a\| \quad \text { for every } x \in X .
$$

In this case, we will say that a realizes $\tau$ in $\widehat{X}$.

Let $X$ be a normed space. If the type $\tau$ is realized in $X$, say, if $\tau=\tau_{a}$, then for any $r>0$, the set

$$
\tau^{-1}[0, r]
$$

is the ball $\{x \in X \mid\|x+a\| \leq r\}$. Now, if $\tau$ is realized by an element $a \in \widehat{X}$, where $\widehat{X}$ is an ultrapower of $X$, the set (1) is the intersection of $X$ with the ball $\{x \in \widehat{X} \mid\|x+a\| \leq r\}$. It is then natural to ask whether (1) can be approximated by balls in $X$; if so, it is also natural to ask whether the radius of these balls can be taken to be $r$, and even whether the norm of their centers can be taken to be $\tau(0)$. In this paper we show that all of these approximation properties in fact characterize Banach space stability.

Let $X$ be a normed space. If $\tau \in \mathcal{T}(X)$, let us say that $\tau$ is approximable if for every $r>0$ and every $\varepsilon>0$, the set $\tau^{-1}[0, r]$ is within $\varepsilon$ of a set formed by finite unions and intersections of balls in $X$. (See Definition 2.2.) Let us say that $\tau$ is strongly approximable if $\tau$ is approximable and the radii of the balls approximating $\tau^{-1}[0, r]$ can be taken arbitrarily close to $r$, and the norm of their centers arbitrarily close to the norm of $\tau$. In Theorem 4.1, we prove that the following conditions are equivalent for a separable Banach space $X$.

1. $X$ is stable;

2. Every type on $X$ is approximable;

3. Every type on $X$ is strongly approximable.

By definition, every type on $X$ is a pointwise limit of types realized in $X$. Thus, if $X$ is separable, $\mathcal{T}(X)$ is separable with respect to the topology of pointwise convergence. It is a well-known fact that if $X$ stable, then $\mathcal{T}(X)$ is strongly separable, i.e., separable with respect to the topology of uniform convergence on bounded subsets of $X$. The converse was proved to be false by E. Odell (see $[6,8]$ ). The preceding theorem explains to what extent stability of $X$ is equivalent to approximability of types on $X$ by types realized in $X$. 
In Proposition 3.1, we characterize approximable functions in terms of finite representability: Let $f$ be a real-valued function on $X$ which is uniformly continuous on every bounded subset of $X$. Then the following conditions are equivalent.

1. $f$ is approximable;

2. Whenever $Y$ is finitely represented in $X$, there is a unique real-valued function $g$ on $Y$ such that $(Y, g)$ is finitely represented in $(X, f)$.

The proofs are based on ideas from model theory. Proposition 2.6 is inspired by the "Definability of Types" lemma in [7].

We will make heavy use of Banach space ultrapowers. For an introduction, we refer the reader to [3].

Throughout the paper, $X$ denotes a normed space. If $M>0$, we denote by $B(M)$ the set of elements of $X$ of norm at most $M$.

2. Constructible sets and approximable types. Let us first recall that a positive boolean combination of the sets $S_{1}, \ldots, S_{n}$ is a set obtained from $S_{1}, \ldots, S_{n}$ by taking finite unions and intersections.

2.1. Definition. Let $X$ be a normed space. A construction $C$ in $X$ is a positive boolean combination of sets of the form

$$
\left\{x \in X \mid\left\|x+a_{i}\right\| \in I_{i}\right\}, \quad a_{1}, \ldots, a_{n} \in X .
$$

We write $C=C\left(a_{1}, \ldots, a_{n} ; I_{1}, \ldots, I_{n}\right)$. If $I_{1}, \ldots, I_{n}=I$, we write $C=$ $C\left(a_{1}, \ldots, a_{n} ; I\right)$.

If $C\left(a_{1}, \ldots, a_{n} ; I_{1}, \ldots, I_{n}\right)$ is a construction in $X$, we denote by

$$
\left[C\left(a_{1}, \ldots, a_{n} ; I_{1}, \ldots, I_{n}\right)\right]
$$

the subset of $X$ determined by $C$. We will call a subset $X$ constructible if it is of the form (2). If $a_{1}, \ldots, a_{n}$ are in a given subset $A$ of $X$, we say that the set (2) is constructible over $A$.

Thus, the class of constructible subsets of $X$ is the ring generated by the balls in $X$.

2.2. Definition. Let $X$ be a normed space and let $f$ be a real-valued function on $X$. We say that $f$ is approximable if the following condition holds. For every choice of $M, \varepsilon>0$ and every interval $I$ there exist a construction $C\left(a_{1}, \ldots, a_{n} ; J\right)$ and $\delta>0$ such that

1. $B(M) \cap f^{-1}[I] \subseteq\left[C\left(a_{1}, \ldots, a_{n} ; J\right)\right]$;

2. $B(M) \cap\left[C\left(a_{1}, \ldots, a_{n} ; J+[-\delta, \delta]\right)\right] \subseteq f^{-1}[I+[-\varepsilon, \varepsilon]]$.

If, regardless of the choice of $M$ and $\varepsilon$, the set $C$ can always be chosen constructible over a given subset $A$ of $X$, we say that $f$ is approximable over $A$. 
We will express the fact that the inclusions in 1 and 2 hold by saying that $\left[C\left(a_{1}, \ldots, a_{n} ; J\right)\right]$ is $(\varepsilon, \delta)$-equivalent to $f^{-1}[I]$ in the ball $B(M)$.

Notice that if $f: X \rightarrow \mathbb{R}$ is approximable, then it is approximable over any given dense subset of $X$.

2.3. Proposition. Let $X$ be a normed space and let $f$ be a real-valued function on $X$. The following conditions are equivalent.

1. $f$ is constructible over $A$;

2. For every $M, \varepsilon>0$ and every interval I of the form $[\alpha, \infty)$ there exist a construction $C\left(a_{1}, \ldots, a_{n} ; J\right)$ with $a_{1}, \ldots, a_{n} \in A$ and $\delta>0$ such that $\left[C\left(a_{1}, \ldots, a_{n} ; J\right)\right]$ is $(\varepsilon, \delta)$-equivalent to $f^{-1}[I]$ in $B(M)$;

3. For every $M, \varepsilon>0$ and every interval I of the form $(\alpha, \infty)$ there exist a construction $C\left(a_{1}, \ldots, a_{n} ; J\right)$ with $a_{1}, \ldots, a_{n} \in A$ and $\delta>0$ such that $\left[C\left(a_{1}, \ldots, a_{n} ; J\right)\right]$ is $(\varepsilon, \delta)$-equivalent to $f^{-1}[I]$ in $B(M)$;

4. For every $M, \varepsilon>0$ and every interval $I$ of the form $(-\infty, \alpha]$ there exist a construction $C\left(a_{1}, \ldots, a_{n} ; J\right)$ with $a_{1}, \ldots, a_{n} \in A$ and $\delta>0$ such that $\left[C\left(a_{1}, \ldots, a_{n} ; J\right)\right]$ is $(\varepsilon, \delta)$-equivalent to $f^{-1}[I]$ in $B(M)$.

P r o of. The equivalence $2 \Leftrightarrow 3$ is immediate, the equivalence $3 \Leftrightarrow 4$ follows by taking complements, and the implication $3 \& 4 \Rightarrow 1$ is proved by taking intersections.

Now we focus on a particular kind of real-valued functions, namely, types.

2.4. Definition. Let $X$ be a normed space and let $\tau: X \rightarrow \mathbb{R}$ be a type on $X$. We will say that $\tau$ is strongly approximable if

- $\tau$ is approximable;

- The interval $J$ of Definition 2.2 can always be taken arbitrarily close to $I$, and the norm of $a_{1}, \ldots, a_{n}$ can be chosen arbitrarily close to the norm of $\tau$.

2.5. Proposition. Let $X$ be a normed space and let $\tau$ be a type on $X$. The following conditions are equivalent.

1. $\tau$ is strongly approximable;

2. For every $M, \varepsilon>0$ and every interval of the form $[0, \alpha]$ there exist a construction $C\left(a_{1}, \ldots, a_{n} ;[0, \beta]\right)$ and $\delta>0$ such that

(i) $\left[C\left(a_{1}, \ldots, a_{n} ;[0, \beta]\right)\right]$ is $(\varepsilon, \delta)$-equivalent to $\tau^{-1}[0, \alpha]$ in $B(M)$;

(ii) $|\beta-\alpha|<\varepsilon$ and $\mid\left\|a_{i}\right\|-\tau(0) \|<\varepsilon$ for $i=1, \ldots, n$.

Pr o of. Immediate from Definition 2.4 and $1 \Leftrightarrow 4$ of Proposition 2.3.

2.6. Proposition. Suppose that $X$ is a stable Banach space. Then every type on $X$ is strongly approximable.

Proof. Let $\tau \in X$. Take $M, \varepsilon>0$ and an interval $[0, \alpha]$. We will define a construction $C\left(d_{1}, \ldots, d_{r} ;[0, \beta]\right)$ and $\delta>0$ such that 
(I) $B(M) \cap \tau^{-1}[0, \alpha] \subseteq\left[C\left(d_{1}, \ldots, d_{r} ;[0, \beta]\right)\right]$;

(II) $B(M) \cap\left[C\left(d_{1}, \ldots, d_{r} ;[0, \beta+\delta]\right)\right] \subseteq \tau^{-1}[0, \alpha+\varepsilon]$.

Take $\beta$ and $\delta$ such that

$$
\alpha<\beta<\beta+\delta<\alpha+\varepsilon .
$$

Without loss of generality, we can take $\delta$ such that

$$
\delta<\min \{\beta-\alpha,(\alpha+\varepsilon)-(\beta+\delta)\} .
$$

Take also positive numbers $\eta, \eta_{0}, \eta_{1}, \ldots$ such that

$$
\delta<\eta_{0}<\eta_{1}<\ldots<\eta
$$

and $\eta$ is less than the minimum in (3).

We will now construct, inductively,

- A sequence $a_{0}, a_{1}, \ldots$ in $B(\tau(0)+\varepsilon)$;

- For $i=-1,0,1,2, \ldots$, sets $S(i), T(i)$ of subsets of $\{0, \ldots, i\}$;

- Elements $u_{i+1}^{s} \in B(M)$ for $s \in S(i)$ and $v_{i+1}^{t} \in B(M)$ for $t \in T(i)$.

Suppose that we have defined $a_{0}, a_{1}, \ldots, a_{n}, S(-1), \ldots, S(n-1)$, $T(-1), \ldots, T(n-1)$, and $u_{i}^{s}, v_{i}^{t}$ for $i=0, \ldots, n$ and $s \in S(i), t \in T(i)$. We now define the sets $S(n), T(n)$ and the elements $u_{i+1}^{s}, v_{i+1}^{t}$.

Let

$$
S(n)=\left\{s \subseteq\{0, \ldots, n\} \mid B(M) \cap \tau^{-1}\left[0, \alpha+\eta_{n}\right] \cap \bigcap_{i \in s} \tau_{a_{i}}^{-1}[\beta, \infty) \neq \emptyset\right\} .
$$

For each $s \in S(n)$, let $u_{n+1}^{s}$ be an element of $X$ such that

Similarly, let

$$
u_{n+1}^{s} \in B(M) \cap \tau^{-1}\left[0, \alpha+\eta_{n}\right] \cap \bigcap_{i \in s} \tau_{a_{i}}^{-1}[\beta, \infty) .
$$

$$
T(n)=\left\{t \subseteq\{0, \ldots, n\} \mid B(M) \cap \tau^{-1}\left[\alpha+\varepsilon-\eta_{n}, \infty\right) \cap \bigcap_{i \in t} \tau_{a_{i}}^{-1}[0, \beta+\delta] \neq \emptyset\right\},
$$

and for each $t \in T(n)$ let $v_{n+1}^{t}$ be an element of $X$ such that

We now define $a_{n+1}$. Let

$$
v_{n+1}^{t} \in B(M) \cap \tau^{-1}\left[\beta+\varepsilon-\eta_{n}, \infty\right) \cap \bigcap_{i \in t} \tau_{a_{i}}^{-1}[0, \beta+\delta] .
$$

$$
F=\left\{u_{i+1}^{s} \mid-1 \leq i \leq n, s \in S(i)\right\} \cup\left\{v_{i+1}^{t} \mid-1 \leq i \leq n, t \in T(i)\right\} .
$$

Since $F$ is finite, there exists $a \in F \cap B(\tau(0)+\varepsilon)$ such that

$$
\begin{array}{rlll}
x \in F \cap \tau^{-1}\left[0, \alpha+\eta_{n}\right] & \text { implies } & \|a+x\| \in\left[0, \alpha+\eta_{n+1}\right], \\
\left.x \in F \cap \tau^{-1}\left[\alpha+\varepsilon-\eta_{n}, \infty\right)\right] & \text { implies } & \|a+x\| \in\left[\alpha+\varepsilon-\eta_{n+1}, \infty\right) .
\end{array}
$$

Let $a_{n+1}$ be such an element $a$. 
2.7. Claim. Suppose that $0 \leq i \leq n$ and $s \in S(i-1), t \in T(i-1)$. Then

$$
\left\|a_{n}+u_{i}^{s}\right\| \in\left[0, \alpha+\eta_{n}\right] \quad \text { and } \quad\left\|a_{n}+v_{i}^{t}\right\| \in\left[\alpha+\varepsilon-\eta_{n}, \infty\right) .
$$

Claim 2.7 follows immediately from the preceding definitions.

2.8. Claim. Suppose that $0 \leq i(0)<i(1)<\ldots<i(n)$ and

$$
\left.B(M) \cap \tau^{-1}[0, \alpha] \cap \bigcap_{j=0}^{n} \tau_{a_{i(j)}}^{-1}[\beta, \infty)\right] \neq \emptyset .
$$

Then there exist $b_{0}, \ldots, b_{n} \in B(M)$ such that

$$
\begin{array}{ll}
\left\|a_{i(j)}+b_{k}\right\| \in[\beta, \infty) & \text { for } 0 \leq j<k \leq n, \\
\left\|a_{i(j)}+b_{k}\right\| \in[0, \alpha+\eta] & \text { for } 0 \leq k \leq j \leq n .
\end{array}
$$

Proof. Inductively, we construct $b_{0}, \ldots, b_{n}$ such that

$$
\begin{aligned}
& \left\|a_{i(j)}+b_{k}\right\| \in[\beta, \infty) \quad \text { for } 0 \leq j<k \leq n \text {, } \\
& \left\|a_{i(j)}+b_{k}\right\| \in\left[0, \alpha+\eta_{i(j)}\right] \quad \text { for } 0 \leq k \leq j \leq n .
\end{aligned}
$$

First we note that $S(i(0)-1) \neq \emptyset$; in fact, $\emptyset \in S(i(0)-1)$ since

$$
B(M) \cap \tau^{-1}\left[0, \alpha+\eta_{i(0)}\right] \supseteq B(M) \cap \tau^{-1}[0, \alpha] \neq \emptyset .
$$

Take $s \in S(i(0))$ and let $b_{0}$ be $u_{i(0)}^{s}$. Then, by Claim 2.7 above, we have

$$
\left\|a_{i(j)}+b_{0}\right\| \in\left[0, \alpha+\eta_{i(j)}\right] \quad \text { for } 0 \leq j \leq n .
$$

Assume that we have $b_{1}, \ldots, b_{k}$ as desired. Let $s=\{i(0), \ldots, i(k)\}$. From the definition of $S(i(k))$, we must have $s \in S(i(k))$. Let $b_{k+1}$ be $u_{k+1}^{s}$. Then

$$
\left.\left\|a_{i(j)}+b_{k+1}\right\| \in[\beta, \infty)\right] \quad \text { for } 0 \leq j \leq k,
$$

and by Claim 2.7,

$$
\left\|a_{i(j+1)}+b_{k+1}\right\| \in\left[0, \alpha+\eta_{i(j+1)}\right] \quad \text { for } 0 \leq k \leq j \leq n-1 .
$$

We have proved Claim 2.8.

2.9. Claim. Suppose that $0 \leq i(0)<i(1)<\ldots<i(n)$ and

$$
\left.B(M) \cap \tau^{-1}[\alpha+\varepsilon, \infty)\right] \cap \bigcap_{j=0}^{n} \tau_{a_{i(j)}}^{-1}[0, \beta+\delta] \neq \emptyset .
$$

Then there exist $c_{1}, \ldots, c_{n} \in B(M)$ such that

$$
\begin{aligned}
& \left\|a_{i(j)}+c_{k}\right\| \in[0, \beta+\delta] \quad \text { for } 0 \leq j<k \leq n \text {, } \\
& \left\|a_{i(j)}+c_{k}\right\| \in[\alpha+\varepsilon-\eta, \infty) \quad \text { for } 0 \leq k \leq j \leq n \text {. }
\end{aligned}
$$


Proof. The proof is analogous to that of Claim 2.8. We construct $c_{1}, \ldots, c_{n}$ inductively such that

$$
\begin{aligned}
\left\|a_{i(j)}+c_{k}\right\| & \in[0, \beta+\delta] & & \text { for } 0 \leq j<k \leq n, \\
\left\|a_{i(j)}+c_{k}\right\| & \in\left[\alpha+\varepsilon-\eta_{i(j)}, \infty\right) & & \text { for } 0 \leq k \leq j \leq n .
\end{aligned}
$$

2.10. Claim. There exists a number $N \in \mathbb{N}$ with the following property. Whenever $0 \leq i(0)<\ldots<i(N) \leq 2 N$,

(i) There does not exist a sequence $\left(b_{k}\right)_{0 \leq k \leq N}$ in $B(M+\tau(0)+\varepsilon)$ satisfying

$$
\begin{array}{ll}
\left\|a_{i(j)}+b_{k}\right\| \in[\beta, \infty) & \text { for } 0 \leq j<k \leq N, \\
\left\|a_{i(j)}+b_{k}\right\| \in[0, \alpha+\eta] & \text { for } 0 \leq k \leq j \leq N
\end{array}
$$

(ii) There does not exist a sequence $\left(c_{k}\right)_{0 \leq k \leq N}$ in $B(M+\tau(0)+\varepsilon)$ satisfying

$$
\begin{array}{ll}
\left\|a_{i(j)}+c_{k}\right\| \in[0, \beta+\delta] & \text { for } 0 \leq j \leq k \leq N, \\
\left\|a_{i(j)}+c_{k}\right\| \in[\alpha+\varepsilon-\eta, \infty) & \text { for } 0 \leq k<j \leq N .
\end{array}
$$

Pr o o f. Suppose that the claim is false. Then, for arbitrarily large $N \in \mathbb{N}$ there will be $0 \leq i(0)<\ldots<i(N) \leq 2 N$ and either a sequence $\left(b_{k}\right)_{0 \leq k \leq N}$ in $B(M+\tau(0)+\varepsilon)$ such that (4) holds, or $\left(c_{k}\right)_{0 \leq k \leq N}$ in $B(M+\tau(0)+\varepsilon)$ such that (5) holds. Now, for any given $N$ there are finitely many choices for $0 \leq i(0)<\ldots<i(N) \leq 2 N$. Hence, König's lemma provides a subsequence $\left(a_{n(l)}\right)_{l \in \mathbb{N}}$ of $\left(a_{n}\right)$ and either a sequence $\left(b_{k}\right)_{k \in \mathbb{N}}$ in $B(M+\tau(0)+\varepsilon)$ such that

$$
\begin{aligned}
\left\|a_{i(l)}+b_{k}\right\| & \in[\beta, \infty) & & \text { for } 0 \leq l<k, \\
\left\|a_{i(l)}+b_{k}\right\| & \in[0, \alpha+\eta] & & \text { for } 0 \leq k \leq l,
\end{aligned}
$$

or a sequence $\left(c_{k}\right)_{k \in \mathbb{N}}$ in $B(M+\tau(0)+\varepsilon)$ such that

$$
\begin{aligned}
\left\|a_{i(l)}+c_{k}\right\| & \in[0, \beta+\delta] & & \text { for } 0 \leq l \leq k, \\
\left\|a_{i(l)}+c_{k}\right\| & \in[\alpha+\varepsilon-\eta, \infty) & & \text { for } 0 \leq k<l .
\end{aligned}
$$

Either case contradicts the stability of $X$. Claim 2.10 is proved.

Fix $N$ as in Claim 2.10. Define

$$
\left\{d_{1}, \ldots, d_{r}\right\}=\left\{a_{i(j)} \mid 0 \leq i(0)<\ldots<i(N) \leq 2 N, 0 \leq j \leq N\right\}
$$

and

$$
C\left(d_{1}, \ldots, d_{r} ;[0, \beta]\right)=\bigcup_{0 \leq i(0)<\ldots<i(N) \leq 2 N} \bigcap_{0 \leq j \leq N} \tau_{a_{i(j)}}[0, \beta] .
$$

Condition (II) follows directly from Claim 2.9 and the choice of $N$. To prove (I), suppose that $x \in B(M)$ and $x \notin[C]$. Fix one of the intersections in (6). The element $x$ is not in this intersection, so there exists an index $i\left(j_{0}\right)$ 
such that $x \notin \tau_{a_{i\left(j_{0}\right)}}[0, \beta]$. Now take an $N$-element subset of $\{1, \ldots, 2 N\}$ not containing $a_{i\left(j_{0}\right)}$ and consider the intersection corresponding to this set in (6). Repeat the argument to find $i\left(j_{1}\right)$ distinct from $i\left(j_{0}\right)$ such that $x \notin \tau_{a_{i\left(j_{1}\right)}}[0, \beta]$. The argument can be iterated $N$ times. But then Claim 2.8 and the choice of $N$ imply $x \notin \tau^{-1}[0, \alpha]$.

REMARK. It is well known that the space of types of a stable Banach space is strongly separable, i.e., separable with respect to the topology of uniform convergence on bounded sets. (The converse is not true; see $[6,8]$.) This is immediate from Proposition 2.6. In fact, it is easy to see that if every type on $X$ is approximable, then the density of $\mathcal{T}(X)$ with respect to the strong topology must equal the density of $X$ (with respect to the norm topology).

3. Approximable functions. Let $X$ be a normed space and let $f$ be a real-valued function on $X$ which is uniformly continuous on every bounded subset of $X$. An ultrapower of $(X, f)$ is defined as follows. If $\mathcal{U}$ is an ultrafilter, then $(\widehat{X}, \widehat{f})$ is the ultrapower of $(X, f)$ with respect to $\mathcal{U}$ if

- $\widehat{X}$ is the ultrapower of $X$ with respect to $\mathcal{U}$;

- Whenever $x \in \widehat{X}$ and $\left(x_{i}\right)_{i \in I}$ is a representative of $x$ in $\widehat{X}$, we have $\widehat{f}(x)=\lim _{\mathcal{U}}\left(x_{i}\right)_{i \in I}$.

The fact that $f$ is uniformly continuous on bounded subsets of $X$ ensures that $\widehat{f}$ is well defined.

An ultrapower $(\widehat{X}, \widehat{f})$ of $(X, f)$ has the property that it is finitely represented in $(X, f)$. This means that whenever $E$ is a finite-dimensional subspace of $\widehat{X}$ and $M, \varepsilon>0$, there exists a finite-dimensional subspace $F$ of $X$ such that $(E, \widehat{f}\lceil E)$ and $(F, f\lceil E)$ are $(1+\varepsilon)$-isomorphic in the sense that there exists a $(1+\varepsilon)$-isomorphism $\varphi: E \rightarrow F$ satisfying $|f(\varphi(x))-\widehat{f}(x)| \leq \varepsilon$ for every $x \in E$ of norm at most $M$.

Let $X$ and $Y$ be normed spaces containing a common subset $A$. If $\varepsilon>0$, we say that $X$ and $Y$ are $(1+\varepsilon)$-isomorphic over $A$ if there exists a $(1+\varepsilon)$ isomorphism $\varphi: X \rightarrow Y$ such that $\varphi \nmid A$ is the identity. We will say that $Y$ is $A$-finitely represented in $X$ if the following condition holds. Given $\varepsilon>0$ and a finite-dimensional subspace $F$ of $Y$, there exists a subspace $E$ of $X$ such that the spaces $\overline{\operatorname{span}}[E \cup A]$ and $\overline{\operatorname{span}}[F \cup A]$ are $(1+\varepsilon)$-isomorphic over $A$.

We will now characterize approximability of real-valued functions in terms of finite representability. Let us first notice the following.

Remarks. 1. If $X$ and $Y$ contain a common subset $A$ and $Y$ is $A$ finitely represented in $X$, then there is an ultrapower $(\widehat{X}, \widehat{f})$ of $(X, f)$ and an embedding $\varphi: Y \rightarrow \widehat{X}$ which fixes $A$ pointwise. 
2. If $(\widehat{X}, \widehat{f})$ is an ultrapower of $(X, f)$ and $f$ is approximable over $A$, then so is $\widehat{f}$; in fact, if $0<M<M^{\prime}, 0<\varepsilon<\varepsilon^{\prime}<\varepsilon^{\prime \prime}$, and $0<\delta<\delta^{\prime}<\delta^{\prime \prime}$ are such that $\left[C\left(a_{1}, \ldots, a_{n} ; J\right)\right]_{X}$ is $\left(\varepsilon^{\prime}-\varepsilon, \delta^{\prime \prime}\right)$-equivalent to $f^{-1}[I+[-\varepsilon, \varepsilon]]$ in the ball $B_{X}\left(M^{\prime}\right)$, then $\left[C\left(a_{1}, \ldots, a_{n} ; J+[-\delta, \delta]\right]_{\widehat{X}}\right.$ is $\left(\varepsilon^{\prime \prime}, \delta^{\prime}-\delta\right)$-equivalent to $\widehat{f}^{-1}[I]$ in the ball $B_{\widehat{X}}(M)$.

3.1. Proposition. Let $X$ be a normed space and let $f$ be a real-valued function on $X$ which is uniformly continuous on every bounded subset of $X$. Then, if $A$ is a subset of $X$, the following conditions are equivalent.

1. $f$ is approximable over $A$;

2. Whenever $Y \supseteq A$ and $Y$ is A-finitely represented in $X$, there is a unique real-valued function $g$ on $Y$ such that $(Y, g)$ is A-finitely represented in $(X, f)$.

Pr o of. $1 \Rightarrow 2$ follows easily from the preceding remarks. We prove $2 \Rightarrow 1$.

Suppose that $f$ is not approximable over $A$. Take $M, \varepsilon>0$ and an interval $I$ such that there do not exist $\left[C\left(a_{1}, \ldots, a_{n} ; J\right)\right]$ with $a_{1}, \ldots, a_{n} \in A$ and $\delta>0$ with $\left[C\left(a_{1}, \ldots, a_{n} ; J\right)\right](\varepsilon, \delta)$-equivalent to $f^{-1}[I]$ in the ball $B(M)$. Without loss of generality, we can assume that $I$ is bounded.

Let

$$
\begin{aligned}
\mathfrak{C}=\left\{C\left(a_{1}, \ldots, a_{n} ; J\right) \mid a_{1}, \ldots, a_{n} \in A\right. & \text { and } \\
B(M) & \left.\cap f^{-1}[I] \subseteq\left[C\left(a_{1}, \ldots, a_{n} ; J\right)\right]\right\} .
\end{aligned}
$$

By our assumption, whenever $C\left(a_{1}, \ldots, a_{n} ; J\right) \in \mathfrak{C}$ and $\delta>0$,

$$
B(M) \cap\left(\left[C\left(a_{1}, \ldots, a_{n} ; J+[-\delta, \delta]\right)\right] \cap \complement f^{-1}[I+[-\varepsilon, \varepsilon]]\right) \neq \emptyset .
$$

Also, $\mathfrak{C}$ is closed under finite intersections. Hence, there exists an ultrapower $(\widehat{X}, \widehat{f})$ of $(X, f)$ and $b \in \widehat{X}$ such that

$$
b \in B(M) \cap \bigcap_{C\left(a_{1}, \ldots, a_{n} ; J\right) \in \mathfrak{C}}\left[C\left(a_{1}, \ldots, a_{n} ; J\right)\right] \cap \complement \widehat{f}^{-1}[I+[-\varepsilon / 2, \varepsilon / 2]] .
$$

Now, notice that if $a_{1}, \ldots, a_{n} \in A$ and $b \in\left[C\left(a_{1}, \ldots, a_{n} ;(-\infty, \alpha]\right)\right]$, then for every $\beta>\alpha$ we must have

$$
B(M) \cap f^{-1}(I) \cap\left[C\left(a_{1}, \ldots, a_{n} ;(-\infty, \beta]\right)\right] \neq \emptyset
$$

(otherwise, $\left[C\left(a_{1}, \ldots, a_{n} ;[\beta, \infty)\right)\right] \in \mathfrak{C}$ and $b \in\left[C\left(a_{1}, \ldots, a_{n} ;[\beta, \infty)\right)\right]$, which is impossible). Hence, there exists an ultrapower $\left(\widehat{X}^{\prime}, \widehat{f}^{\prime}\right)$ of $(X, f)$ and $b^{\prime} \in$ $\widehat{X}^{\prime}$ such that

(i) $\widehat{f}^{\prime}\left(b^{\prime}\right) \in I$;

(ii) $b^{\prime} \in\left[C\left(a_{1}, \ldots, a_{n} ;(-\infty, \alpha]\right)\right]$ whenever $a_{1}, \ldots, a_{n} \in A$ and $b \in$ $\left[C\left(a_{1}, \ldots, a_{n} ;(-\infty, \alpha]\right)\right]$. 
By (ii), there is an isometry between $\overline{\operatorname{span}}[\{b\} \cup A]$ and $\overline{\operatorname{span}}\left[\left\{b^{\prime}\right\} \cup A\right]$ mapping $b$ to $b^{\prime}$ and fixing $A$ pointwise. But $\overline{\operatorname{span}}[\{b\} \cup A]$ and $\overline{\operatorname{span}}\left[\left\{b^{\prime}\right\} \cup A\right]$ are $A$-finitely represented in $X$ and $\widehat{f}(b) \notin I$, so we are in contradiction with condition 2.

4. Approximable types and stability. We now prove the main result.

4.1. Theorem. Let $X$ be a separable Banach space. Then the following conditions are equivalent.

1. $X$ is stable;

2. Every type on $X$ is approximable;

3. Every type on $X$ is strongly approximable.

Proof. $1 \Rightarrow 3$ is Proposition 2.6. We prove $2 \Rightarrow 1$.

Suppose that $X$ is not stable. Then there exist bounded sequences $\left(a_{m}\right)$ and $\left(b_{n}\right)$ in $X$ and real numbers $\alpha, \beta$ such that

$$
\sup _{m<n}\left\|a_{m}+b_{n}\right\| \leq \alpha<\beta \leq \inf _{n<m}\left\|a_{m}+b_{n}\right\| .
$$

Without loss of generality, we can assume that $\left(a_{m}\right)$ is type determining, i.e., there exists a type $\tau \in \mathcal{T}(X)$ such that $\tau(x)=\lim _{m \rightarrow \infty}\left\|a_{m}+x\right\|$ for every $x \in X$.

By (7) there exists an ultrapower $\widehat{X}$ of $X$, an element $a \in \widehat{X}$, and types $\varrho_{1}, \varrho_{2}$ on $\widehat{X}$ such that

- $\left(\widehat{X}, \varrho_{1}\right)$ and $\left(\widehat{X}, \varrho_{2}\right)$ are finitely represented in $(X, \tau)$;

- $\varrho_{1}(a) \leq \alpha$ and $\varrho_{2}(a) \leq \beta$.

But then $\tau$ cannot be approximable, by Proposition 3.1.

REMARK. The concepts considered here are particularizations of concepts from the logical analysis of stability in [4]. Indeed, the notions of type, constructible subset, and approximable function correspond (respectively) to the "quantifier-free" versions of the notions of type, definable subset, and definable real-valued relation considered in [4].

\section{References}

[1] D. Aldous, Subspaces of $L_{1}$ via random measures, Trans. Amer. Math. Soc. 267 (1981), 445-463.

[2] S. Guerre-Delabrière, Classical Sequences in Banach Spaces, Marcel Dekker, New York, 1992.

[3] S. Heinrich, Ultraproducts in Banach space theory, J. Reine Angew. Math. 313 (1980), 72-104.

[4] J. Iovino, Stable theories in functional analysis, $\mathrm{PhD}$ thesis, Univ. of Illinois at Urbana-Champaign, 1994. 
[5] J.-L. Krivine et B. Maurey, Espaces de Banach stables, Israel J. Math. 39 (1981), 273-295.

[6] E. Odell, On the types in Tsirelson's space, in: Longhorn Notes, Texas Functional Analysis Seminar, 1982-1983.

[7] A. Pillay, Geometric Stability Theory, Clarendon Press, Oxford, 1996.

[8] Y. Raynaud, Stabilité et séparabilité de l'espace des types d'un espace de Banach: Quelques exemples, in: Séminarie de Géométrie des Espaces de Banach, Paris VII, Tome II, 1983.

Department of Mathematical Sciences

Carnegie Mellon University

Pittsburgh, Pennsylvania 15213

U.S.A.

E-mail: iovino@cmu.edu 